\title{
10 COMPORTAMENTO AUTOLESIVO AO LONGO DO CICLO VITAL: REVISÃO INTEGRATIVA DA LITERATURA ${ }^{1}$
}

\author{
| Aline Conceição Silva²; Nadja Cristiane Lappann Botti ${ }^{3} \mid$
}

\section{RESUMO}

CONTEXTO: O comportamento autolesivo deliberado é caracterizado pelo comportamento de autodano intencional e sem intenção consciente de suicídio. Permeia todo ciclo vital, sendo mais prevalente na adolescência, principalmente no sexo feminino. O fenômeno está relacionado com mecanismos adaptativos mal elaborados e outras circunstâncias de vida.

OBJETIVO: Identificar na literatura científica os fatores associados ao comportamento autolesivo deliberado no ciclo vital.

MÉTODOS: Realizado estudo de revisão integrativa da literatura, sendo utilizada como base de dados a Biblioteca Virtual em Saúde. Foram utilizados como termos de busca self harm AND fator de risco, sendo encontrados 173 artigos. De acordo com critérios de inclusão foram elegíveis 23 artigos para compor o estudo.

RESULTADOS: Após leitura e análise crítica dos artigos emergiram duas categorias de análise: conceito polissêmico da automutilação e automutilação ao longo do ciclo vital. Verificou-se a existência de vários termos sobre o fenômeno e diversas definições. Quanto aos fatores de risco para o comportamento autolesivo identificou-se generalidades no ciclo vital, bem como fatores específicos para cada etapa. Ainda, observou-se a necessidade de estudos sobre os fatores protetores presentes na automutilação.

CONCLUSÃO: O conhecimento sobre os fatores de risco auxilia profissionais e estudiosos na identificação e manejo de casos e futuros estudos sobre automutilação.

PALAVRAS-CHAVE: Automutilação; Fator de risco; Ciclo vital

\section{RESUMEN}

"Conductas autolesivas a lo largo de del ciclo vital: Revisión integradora de la literatura"

CONTEXTO: El comportamiento autolesivo deliberado se caracteriza por el comportamiento de autodano intencional y sin intención consciente de suicidio. Permecia todo ciclo vital, siendo más prevalente en la adolescencia, principalmente en el sexo femenino. El fenómeno está relacionado con mecanismos adaptativos mal elaborados y otras circunstancias de vida.

OBJETIVO: Identificar en la literatura científica los factores asociados al comportamiento autolesivo deliberado en el ciclo vital.

METODOLOGÍA: Realizado estudio de revisión integrativa de la literatura, siendo utilizada como base de datos la Biblioteca Virtual en Salud. Fueron utilizados como términos de búsqueda self harm AND factor de riesgo, siendo encontrados 173 artículos. De acuerdo con criterios de inclusión fueron elegibles 23 artículos para componer el estudio.

RESULTADOS: Después de lectura y análisis crítico de los artículos surgieron dos categorías de análisis: concepto polisémico de la automutilación y automutilación a lo largo del ciclo vital. Se verificó la existencia de varios términos sobre el fenómeno y diversas definiciones. En cuanto a los factores de riesgo para el comportamiento autolesivo se identificó generalidades en el ciclo vital, así como factores específicos para cada etapa. Se observó la necesidad de estudios sobre los factores protectores presentes en la automutilación.

CONCLUSIÓN: El conocimiento sobre los factores de riesgo auxilia a profesionales y estudiosos en la identificación y manejo de casos y futuros estudios sobre automutilación.

\section{DESCRIPTORES: Automutilación; Factor de riesgo; Ciclo vital}

\begin{abstract}
"Self-injurious behavior along the vital cycle: Integrative literature review"

BACKGROUND: Deliberate self-injurious behavior is characterized by intentional self-harm behavior and no conscious intention of suicide. It permeates the entire life cycle, being more prevalent in adolescence, especially in females. The phenomenon is related to poorly elaborated adaptive mechanisms and other life circumstances.

AIM: To identify in the scientific literature the factors associated with deliberate self - injurious behavior in the life cycle.

METHODS: An integrative review of the literature was carried out, and the Virtual Health Library was used as a database. Self harm and risk factor were used as the search terms, and 173 articles were found. According to inclusion criteria, 23 articles were eligible to be included in the study.

RESULTS: After reading and critically analyzing the articles, two categories of analysis emerged: polysemic concept of self-mutilation and self-mutilation throughout the life cycle. There were several terms on the phenomenon and several definitions. Regarding the risk factors for self-injurious behavior, generalities were identified in the life cycle, as well as specific factors for each stage. Furthermore, it was observed the need for studies on the protective factors present in self-mutilation. CONCLUSION: Knowledge about risk factors helps professionals and scholars to identify and manage cases and future studies on self - mutilation.
\end{abstract}

\section{KEYWORDS: Self-mutilation; Risk factor; Life Cycle}

Submetido em 30-03-2016

Aceite em 16-10-2017

1 Artigo retirado da Dissertação de Mestrado "Comportamento autolesivo em redes sociais virtuais”.

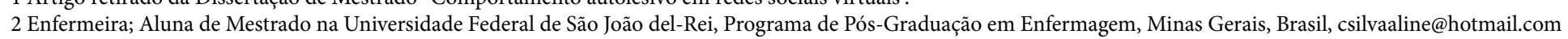


gem, 35501-296 Divinópolis, Minas Gerais, Brasil, nadjaclb@terra.com.br

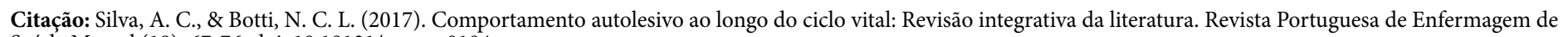
Saúde Mental (18), 67-76. doi: 10.19131/rpesm.0194 


\section{INTRODUÇÃO}

Automutilação deliberada constitui a destruição deliberada direta ou alteração do tecido do corpo sem intenção consciente de suicídio, mas que resulta em ferimentos graves o suficiente para a ocorrência de lesões (Giusti, 2013). Este comportamento está associado a mecanismos mal adaptativos de enfrentamento ou estratégias de regulação de emoção, neste sentido a automutilação deliberada pode ser utilizada para alívio de emoções muito fortes e diminuição da tensão. Além das inequívocas consequências físicas observa-se que a automutilação deliberada está associada com uma variedade e complexidade de interferências negativas como na terapia e relacionamentos interpessoais ou extremos como no caso de resultar, involuntariamente, em morte (Gratz, 2001).

O comportamento autolesivo ocorre em diversas faixas etárias, sendo predominante em adolescentes do sexo feminino (Cedaro \& Nascimento, 2013). Em geral, tem início entre os 13 e 14 anos e pode persistir por 10 ou 15 anos ou ainda, por mais tempo (Giusti, 2013). Em relação à prevalência do comportamento autolesivo nessa faixa etária encontra-se 6,9\% entre estudantes ingleses, $35,8 \%$ entre adolescentes japoneses do sexo masculino e encarcerados e $45 \%$ entre adolescentes americanos. (Hawton, Rodham, Evans \& Weatherall, 2002) No tocante aos adolescentes canadenses de 12 a 16 anos de idade encontra-se prevalência de 13,9\% (Matsumoto et al., 2005) e entre os adolescentes americanos de 13\% a 39\% (Lloyd-Richardson, Perrine, Dierker \& Kelley, 2007). No Brasil, os estudos sobre prevalência do comportamento autolesivo ainda se apresentam incipientes, entretanto verifica-se aumento significativo na busca por esse comportamento (Giusti, 2013).

Os fatores comumente relacionados ao comportamento se dividem em fatores demográficos, sociais e familiares, desordens psiquiátricas, características psicológicas, aspectos neurobiológicos e genéticos e situacionais (Skegg, 2005). Um sistema adaptativo mal elaborado constitui um dos principais fatores relacionados a automutilação e detém importante atenção principalmente por se tratar de característica da adolescência, fase prevalente do comportamento (Skegg, 2005). Neste contexto a automutilação deliberada é um comportamento que cada vez mais desafia a atenção dos pesquisadores e clínicos (Gratz, 2001).

Dessa forma o objetivo do presente estudo é identificar na literatura científica fatores associados ao comportamento autolesivo deliberado.

\section{MÉTODOS}

Realizada Revisão Integrativa da Literatura que se caracteriza pelo seguimento de um método minucioso de procura, seleção e avaliação das pesquisas frente à relevância e validade desejada. (Souza, Silva \& Carvalho, 2011) A revisão integrativa de literatura consiste na mais ampla abordagem metodológica referente às revisões, pois permite a inclusão de estudos experimentais e não-experimentais aliados a dados da literatura teórica e empírica para uma compreensão completa do fenômeno analisado (Souza, Silva \& Carvalho, 2011).

A pergunta elaborada como questão norteadora da revisão integrativa da literatura foi: Quais os fatores relacionados ao comportamento autolesivo? A busca dos estudos primários foi realizada na Biblioteca Virtual em Saúde nas bases de dados Indice Bibliográfico Español de Ciencias de La Salud (IBECS), Avaliações de tecnologias Sanitárias (HTA), Literatura Latino-Americana e do Caribe em Ciências da Saúde (LILACS), Ensaios Clínicos Controlados (CENRTAL) e Literatura Internacional em Ciências da Saúde (MEDLINE). Para compor a estratégia de pesquisa mais sensível, utilizamos um descritor sobre o assunto combinada com uma palavra chave comumente utilizada pelos autores sobre o tema Assim, utilizou-se selfharm AND fatores de risco na técnica booleana de descritores em Ciências da Saúde indexados. Para a revisão definiu-se como critérios de inclusão artigos publicados em inglês, espanhol e português que apresentavam fatores para o comportamento autolesivo deliberado. Como critérios de exclusão foram eleitos: referências que não permitiram acesso gratuito ao texto completo on-line e artigos repetidos. Nesta revisão não ocorreram restrições quanto ao período de publicação dos manuscritos analisados. Portanto após identificar os manuscritos que atendiam os critérios de inclusão e exclusão foram analisados artigos publicados no período de 1996 a 2014.

\section{RESULTADOS}

A partir da busca encontrou-se 173 manuscritos sendo 164 MEDLINE, 05 CENRTAL, 02 LILACS, 01 HTA, 01 IBECS. Dentre os 173 artigos encontrados, 158 possuíam resumo disponível. Destes, apenas 29 estavam dentro do tema proposto para a revisão. Dos 29 artigos foram excluídos 06 artigos (02 foram excluídos por não possuir texto completo disponível, 03 artigos estavam em outro idioma, e 01 artigo em banco de dados repetido), sendo utilizado nesta Revisão Integrativa da Literatura o total de 23 artigos (Quadro 1). Todos os artigos encontrados são publicações na língua inglesa. 
Quadro 1 - Artigos Selecionados por Etapas do Ciclo Vital

\begin{tabular}{|c|c|c|c|}
\hline Título do artigo & Autores/Ano & $\begin{array}{l}\text { Natureza do } \\
\text { estudo }\end{array}$ & $\begin{array}{l}\text { Etapa do ciclo } \\
\text { vital }\end{array}$ \\
\hline $\begin{array}{l}\text { Deliberate self harm in adolescents: self report survey in schools } \\
\text { in England }\end{array}$ & $\begin{array}{l}\text { Hawton, Rodham, Evans \& Weather- } \\
\text { all, } 2002\end{array}$ & Transversal & $\begin{array}{l}\text { Infanto/ } \\
\text { Juvenil }\end{array}$ \\
\hline $\begin{array}{l}\text { Revictimization and Self-Harm in Females Who Experienced } \\
\text { Childhood Sexual Abuse }\end{array}$ & $\begin{array}{l}\text { Noll, Horowitz, Bonanno, Tricketti \& } \\
\text { Putnam, } 2003\end{array}$ & Prospectivo & $\begin{array}{l}\text { Infanto/ } \\
\text { Juvenil }\end{array}$ \\
\hline $\begin{array}{l}\text { Rates, risk factors and methods of self harm among minority } \\
\text { ethnic groups in the UK: a systematic review }\end{array}$ & Bhui, Mckenzie \& Rasul, 2007 & $\begin{array}{l}\text { Revisão } \\
\text { Sistemática }\end{array}$ & $\begin{array}{l}\text { Infanto/Juve- } \\
\text { nil e Adulto }\end{array}$ \\
\hline $\begin{array}{l}\text { The prevalence of self-cutting and other self-harm among 13-to } \\
18 \text {-year-old Finnish adolescents }\end{array}$ & $\begin{array}{l}\text { Laukkanen, Rissanen, Honkalampi, } \\
\text { Kylmã, Tolmunen \& Hintikka, } 2009\end{array}$ & $\begin{array}{l}\text { Caso Con- } \\
\text { trole }\end{array}$ & $\begin{array}{l}\text { Infanto/ } \\
\text { Juvenil }\end{array}$ \\
\hline $\begin{array}{l}\text { Risk factors and correlates of deliberate self-harm behavior: A } \\
\text { systematic review }\end{array}$ & Fliege, Lee, Grim \& Klapp, 2009 & $\begin{array}{l}\text { Revisão Bibli- } \\
\text { ográfica }\end{array}$ & $\begin{array}{l}\text { Infanto/Juve- } \\
\text { nil e Adulto }\end{array}$ \\
\hline $\begin{array}{l}\text { Bullying victimisation, self harm and associated factors in Irish } \\
\text { adolescent boys }\end{array}$ & $\begin{array}{l}\text { McMahon, Reulbach, Keeley, Perry \& } \\
\text { Arensman, } 2010\end{array}$ & Transversal & $\begin{array}{l}\text { Infanto/ } \\
\text { Juvenil }\end{array}$ \\
\hline $\begin{array}{l}\text { Deliberate self-harm and psychological problems in young ado- } \\
\text { lescents: Evidence of a bidirectional relationship in girls }\end{array}$ & $\begin{array}{l}\text { Lundh, Wângby-Lundh \& Bjärehed, } \\
2011\end{array}$ & $\begin{array}{l}\text { Longitudinal } \\
\text { Prospectivo }\end{array}$ & $\begin{array}{l}\text { Infanto/ } \\
\text { Juvenil }\end{array}$ \\
\hline $\begin{array}{l}\text { Bullying victimisation and risk of self harm in early adolescence: } \\
\text { longitudinal cohort study }\end{array}$ & $\begin{array}{l}\text { Fisher, Moffitt, Houts, Belsky, Arse- } \\
\text { neault \& Caspi, } 2012\end{array}$ & $\begin{array}{l}\text { Longitudinal } \\
\text { Coorte }\end{array}$ & $\begin{array}{l}\text { Infanto/ } \\
\text { Juvenil }\end{array}$ \\
\hline $\begin{array}{l}\text { Self-harm in young adolescents ( } 12-16 \text { years): } \\
\text { onset and short-term continuation in a community sample }\end{array}$ & $\begin{array}{l}\text { Stallard, Spears, Montgomery, Phillips } \\
\text { \& Sayal, } 2013\end{array}$ & Randomizado & $\begin{array}{l}\text { Infanto/ } \\
\text { Juvenil }\end{array}$ \\
\hline $\begin{array}{l}\text { Associations of deliberate self-harm with loneliness, self-rated } \\
\text { health and life satisfaction in adolescence: Northern Finland } \\
\text { Birth Cohort } 1986 \text { Study }\end{array}$ & $\begin{array}{l}\text { Rönka, Taanila, Koiranen, Sunnari \& } \\
\text { Rautio, } 2013\end{array}$ & $\begin{array}{l}\text { Longitudinal } \\
\text { Coorte }\end{array}$ & $\begin{array}{l}\text { Infanto/ } \\
\text { Juvenil }\end{array}$ \\
\hline $\begin{array}{l}\text { Clinical and social outcomes of adolescent self harm: population } \\
\text { based birth cohort study }\end{array}$ & $\begin{array}{l}\text { Mars, Heron, Crane, Hawton, Lewis, } \\
\text { Macleod, Tilling \& Gunnel, } 2014\end{array}$ & Coorte & $\begin{array}{l}\text { Infanto/ } \\
\text { Juvenil }\end{array}$ \\
\hline $\begin{array}{l}\text { Deliberate self harm and the Probation Service; an overlooked } \\
\text { public health problem? }\end{array}$ & $\begin{array}{l}\text { Wessely, Akhurst, Brown \& Moss, } \\
1996\end{array}$ & Transversal & Adulto \\
\hline $\begin{array}{l}\text { Is suicide risk taken seriously in heavy drinkers who harm } \\
\text { themselves? }\end{array}$ & Taylor, Cooper \& Appleby, 1999 & Transversal & Adulto \\
\hline $\begin{array}{l}\text { Antidepressant treatment and the risk of fatal and non-fatal self } \\
\text { harm in first episode depression: nested case-control study }\end{array}$ & $\begin{array}{l}\text { Martinez, Rietbrock, Wise, Ashby, } \\
\text { Chick, Moseley, Evans \& Gunnel, } \\
2005\end{array}$ & $\begin{array}{l}\text { Longitudinal } \\
\text { Coorte }\end{array}$ & $\begin{array}{l}\text { Adulto e } \\
\text { Idoso }\end{array}$ \\
\hline $\begin{array}{l}\text { Risk of Harm: Inmates Who Harm Themselves } \\
\text { While in Prison Psychiatric Treatment }\end{array}$ & Young, Justice \& Erdberg, 2006 & Transversal & Adulto \\
\hline $\begin{array}{l}\text { Adverse incidents, patient flow and nursing workforce variables } \\
\text { on acute psychiatric wards: the Tompkins acute ward study }\end{array}$ & $\begin{array}{l}\text { Bowers, Allan, Simpson, Nijman \& } \\
\text { Warren, } 2007\end{array}$ & Transversal & Adulto \\
\hline $\begin{array}{l}\text { Prevalence and Correlates of Self-Injury Among University } \\
\text { Students }\end{array}$ & $\begin{array}{l}\text { Gollust, Eisenberg \& Golberstein, } \\
2008\end{array}$ & Transversal & Adulto \\
\hline $\begin{array}{l}\text { Temperament as a propective predictor of self -injury among } \\
\text { patients with Borderline personality disorder }\end{array}$ & $\begin{array}{l}\text { Chapman, Derbidge, Cooney, Hong } \\
\text { \& Linehan, } 2009\end{array}$ & Transversal & Adulto \\
\hline $\begin{array}{l}\text { Self-injury, psychiatric symptoms, and defense mechanisms: } \\
\text { Findings in an Italian nonclinical sample }\end{array}$ & Sarno, Madeddu \& Gratz, 2010 & Transversal & Adulto \\
\hline Deliberate self-harm in older people revisited & $\begin{array}{l}\text { Lamprecht, Pakrasi, Gash \& Swann, } \\
2005\end{array}$ & $\begin{array}{l}\text { Observacio- } \\
\text { nal }\end{array}$ & Idoso \\
\hline Suicide and deliberate self harm in older Irish adults & $\begin{array}{l}\text { Corcoran, Reulbach, Perry \& Arens- } \\
\text { man, } 2010\end{array}$ & Transversal & Idoso \\
\hline Self-injurious behavior in the nursing home setting & $\begin{array}{l}\text { Mahgoub, Klimstra, Kotbi \& Do- } \\
\text { cherty, } 2011\end{array}$ & $\begin{array}{l}\text { Revisão Bibli- } \\
\text { ográfica }\end{array}$ & Idoso \\
\hline $\begin{array}{l}\text { The association between personality disorder and an act of } \\
\text { deliberate self harm in the older person }\end{array}$ & $\begin{array}{l}\text { Ritchie, King, Nolan, O’Connor, Ev- } \\
\text { ans, Toms, Kitchen, Evans, Bielawski, } \\
\text { Lee, \& Blanchard, } 2011\end{array}$ & $\begin{array}{l}\text { Caso Con- } \\
\text { trole }\end{array}$ & Idoso \\
\hline
\end{tabular}

A partir da leitura e análise crítica dos artigos emergiram duas categorias de análise: automutilação conceito polissêmico e automutilação deliberada ao longo do ciclo vital. 


\section{DISCUSSÃO}

\section{Automutilação Conceito Polissêmico}

Encontrado diversidade de conceitos nos artigos referentes à automutilação, entre eles citam-se: automutilação deliberada (Deliberate self harm - DSH) (Mars et al., 2014) (Wessely, Akhurs, Brown \& Moss, 1996), automutilação não fatal (Self Harm - SH) (Bhui, McKenzie \& Rasul, 2007), comportamento de auto dano (Selfinjurious behavior - SIB) (Mahgoub, Klimstra, Kotbi \& Docherty, 2001), auto dano (Self-Injury - SI) (Sarno, Madeddu \& Gratz, 2010) refere-se a qualquer ato deliberado (Wessely et al., 1996) autoinduzido (Noll, Horowitz, Bonanno, Trickett \& Putnam, 2003) (Lundh, Wângby-Lundh \& Bjärehed, 2011) intencional (Lundh et al., 2011) de auto dano (Wessely et al., 1996) (Ronka, Taanila, Koiranen, Sunnari \& Rautio, 2013) (para destruir ou prejudicar o próprio corpo) com um resultado não fatal (Wessely et al., 1996), mas resultando em danos relevantes nos tecidos (Fliege, Lee, Grim \& Klapp, 2009) (Gollust, Elsenberg \& Golberstein, 2008) (Laukkanen et al., 2009). A autolesão é um ato com um resultado não-fatal que o indivíduo faz deliberadamente com a intenção de se prejudicar (Ronka et al., 2013) (Laukkanen et al., 2009) (sem a intervenção de outros (Corcoran, Reulbach, Perry \& Arensman, 2010). No entanto, há definições que incluem outros responsáveis pelo ato prejudicial, neste caso além do próprio indivíduo, o dano pode ser produzido pela família ou tratamento médico que deliberadamente causaram dano físico (Ritchie et al., 2011). Ressalta-se que a automutilação enquanto qualquer ação contra si mesmo apresenta gravidade que necessita de cuidados médicos (Young, Justice \& Erdberg, 2006).

A automutilação também é conhecida como parasuicídio (Wessely et al., 1996), auto envenenamento ou auto lesão (Hawton, Rodham, Evans \& Weatherall, 2002), é muito comum entre adolescentes (Lundh et al., 2011) (Hawton et al., 2002). É um problema que produz sobrecarga nos serviços de saúde (Bhui et al., 2007) e, apesar da dificuldade de se avaliar o grau de intenção suicida, sabe-se que a automutilação é importante fator de risco para o suicídio consumado e para futuro comportamento autolesivo (Bhui et al., 2007). Neste aspecto, encontra-se que a automutilação deliberada enquanto comportamento auto induzido pode apresentar-se sem intenção suicida (Fliege et al., 2009) ou sem consciente intenção suicida (Gollust et al., 2008) (Laukkanen et al., 2009) (McMahona, Reulbach, Keeley, Perry \& Arensman, 2010).
Autolesão ou automutilação deliberada encontra-se, tradicionalmente, ligada a transtornos limítrofes de personalidade, depressão, ansiedade, abuso de substâncias e transtornos alimentares (Gollust et al., 2008). Em geral, o auto dano é considerado uma má adaptação ou estratégia para tentar reduzir, evitar ou regular determinadas emoções avassaladoras para o indivíduo (Sarno et al., 2010).

São comuns entre as pessoas que produzem automutilação deliberada determinadas características, tais como: dificuldade em identificar, entender ou expressar suas emoções, maior frequência de emoções negativas na vida diária, menores recursos pessoal de enfrentamento, baixa autoestima, baixa habilidades de resolução de problemas, menor crença na auto eficácia e maior tendência de auto-culpabilização como forma de enfrentamento. Verificam-se também evidências entre fatores psicopatológicos específicos e o comportamento de autoagressão, tais como desrealização/dissociação e alexitimia (falta de expressividade emocional) (Fliege et al., 2009).

O comportamento de auto dano pode ser direto (por exemplo, auto corte) ou indireto (por exemplo, alimento ou recusa a medicação (Mahgoub et al., 2011). Engloba uma variedade de comportamentos com a intenção de causar um auto dano como produzir um auto corte com quaisquer instrumentos (Ronka et al., 2013) (Laukkanen et al., 2009), mas não inclui tatuagem e bodypiercing (McMahona et al., 2010). Também inclui saltar de altura (McMahona et al., 2010) (Ronka et al., 2013), ingerir medicamento prescrito em excesso ou para além da dosagem terapêutica reconhecida (Ronka et al., 2013) (McCMahona et al., 2010) (Corcoran et al., 2010) ou ingerir drogas lícita ou ilícita em excesso, ingerir uma substância ou objeto não-ingerível (Ronka et al., 2013) (McMahona et al., 2010) altura ou produzir uma queimadura (Ronka et al., 2013). 


\section{Automutilação Deliberada ao Longo do Ciclo Vital}

Elencou-se os fatores de risco para o comportamento autolesivo de acordo com etapas do ciclo vital que se encontram no Quadro 2.

Quadro 2 - Fatores de Risco para o Comportamento

Autolesivo de acordo com Etapas do Ciclo Vital

\begin{tabular}{|c|c|}
\hline Ciclo Vital & Fatores de risco \\
\hline Infanto-juvenil & $\begin{array}{l}\text { Individuais: fatores psicopatológicos especí- } \\
\text { ficos, tais como transtorno de conduta, per- } \\
\text { sonalidade borderline, depressão, ansiedade } \\
\text { e sintomas psicóticos; consumo de álcool e } \\
\text { outras drogas (lícitas e ilícitas); preocupações } \\
\text { com a orientação sexual e níveis de impulsivi- } \\
\text { dade e baixa autoestima; história de compor- } \\
\text { tamento autoagressivo. } \\
\text { Familiares: História familiar de tentativa de } \\
\text { suicídio ou suicídio consumado; história de } \\
\text { maltrato físico por um adulto ou pais; prob- } \\
\text { lema de parentalidade; assédio, abuso físico e } \\
\text { sexual; negligência emocional; separação pre- } \\
\text { coce dos pais, morar com apenas um dos pais; } \\
\text { familiares com história de automutilação. } \\
\text { Sociais: isolamento social; bullying; baixa } \\
\text { escolaridade, formação profissional e/ou em- } \\
\text { prego; amigos com história de automutilação. }\end{array}$ \\
\hline Adultos & $\begin{array}{l}\text { Individuais: associação com transtornos } \\
\text { mentais: depressão, transtorno alimentar, } \\
\text { ansiedade; consumo diário de álcool e outras } \\
\text { drogas (lícitas e ilícitas); tipo de relaciona- } \\
\text { mento; bissexuais e homossexuais femininos; } \\
\text { experiências traumáticas na infância; evento } \\
\text { negativo nos } 06 \text { meses anteriores; dificuldades } \\
\text { de relacionamento com parceiros, amigos, } \\
\text { cônjuges e vizinhos. } \\
\text { Sociais: imigrantes negros; desemprego; crises } \\
\text { financeiras. }\end{array}$ \\
\hline Idosos & $\begin{array}{l}\text { Individuais: Demência, depressão e sintomas } \\
\text { psicóticos; baixa qualidade de vida e saúde } \\
\text { física precária, doença debilitante; história } \\
\text { anterior de automutilação deliberada; de- } \\
\text { pendência alcoólica; } \\
\text { Sociais: idosos institucionalizados }\end{array}$ \\
\hline
\end{tabular}

\section{Automutilação Deliberada na População Infanto- Juvenil}

O estudo de Fliege et al. (2009) evidencia que a automutilação é característica da adolescência, as taxas de automutilação em indivíduos de 12 anos é de 2,7\% entre meninas e 3,1\% entre meninos, sendo que aos 15 anos, as taxas aumentam para $12,6 \%$ para meninas e $4,6 \%$ para meninos. Cerca de $19 \%$ dos pacientes com 16 anos em estudo de coorte de base populacional apresentaram histórico de auto dano na vida, sendo que $40 \%$ dos adolescentes com auto dano apresentaram depressão e transtornos de ansiedade e 35\% com problemas de uso de substâncias (Mars et al., 2014)
O que corrobora com estudo realizado com adolescentes ingleses que evidencia que $13,2 \%$ alunos relatam automutilação deliberada na história da vida (Hawton et al., 2002).

Em relação aos métodos utilizados para infligir as lesões, identificou-se entre meninas de 13-18 anos a prevalência de $11 \%$ de auto corte e $10 \%$ em outros métodos (abuso de drogas lícitas e ilícitas) (Laukkanen et al., 2009). O estudo de Hawton et al (2002) também apresenta alta prevalência para o auto corte $(64,6 \%)$ e intoxicação $(30,7 \%)$, sendo que $12,6 \%$ dos casos necessitaram de tratamento hospitalar. Tem sido sugerido que as meninas expressam através de um comportamento auto prejudicial a sua incapacidade de adaptação e equilíbrio psicológico, neste caso o auto corte é utilizado como método auto terapêutico para alívio de tensão (Laukkanen et al., 2009). Entre os fatores associados à automutilação entre crianças de 5, 7, 10 e 12 anos encontram-se história familiar de tentativa de suicídio ou suicídio consumado e história de maltrato físico por um adulto. Verificam-se também evidências entre fatores psicopatológicos específicos, tais como transtorno de conduta, personalidade borderline, depressão e sintomas psicóticos (Fisher et al., 2012).

Estudo de base populacional investigando a frequência de automutilação deliberada entre adolescentes mostra que $18,0 \%$ das meninas e $5,3 \%$ dos meninos não tinha amigos íntimos, $24,7 \%$ das meninas e $13,7 \%$ dos meninos sentiam que ninguém gostava deles e $40,5 \%$ das meninas e $15,4 \%$ dos meninos estavam insatisfeitos com a própria vida, sendo a solidão um fator importante a ser considerado entre adolescentes (Ronka et al., 2013).

Exposição à violência (psicológica, física ou sexual) também está associada ao comportamento de auto dano. Intimidação frequente ou bullying entre crianças e adolescentes, bem como maus tratos por familiar, problemas de parentalidade e abuso sexual estão implicados em aumento das taxas de automutilação. (Fisher et al., 2012) Investigação sobre maus tratos na infância e automutilação mostram associação entre abuso sexual na infância e posterior automutilação na adolescência mostrando as consequências traumáticas e persistentes de abuso sexual na infância. A incidência de automutilação entre vítimas de abuso sexual é maior mesmo quando comparada com outras formas de maus-tratos infantis (Noll et al., 2003). Adolescentes de 15, 16 e 17 anos com experiência de vitimização de bullying apresentam maior associação com pensamentos e história de automutilação. (McMahona et al., 2010). 
Entre adolescentes de 15-16 anos, para ambos os sexos, observa-se maior associação de automutilação deliberada com relato de aumento de consumo de cigarros ou álcool e número de embriagues (Hawton et al., 2002). Entre adolescentes de 13 a 18 anos observa-se maior risco de auto corte e outros tipos de automutilação entre aqueles que apresentam relato consumo frequente de drogas lícitas e ilícitas (Laukkanen et al., 2009).

Adolescentes com problemas de saúde mental ou uso de substâncias, sem escolaridade, formação profissional e/ou emprego são grupo de alto risco para automutilação. No total, 19\% da amostra relataram história da vida de auto danos na idade de 16 anos; destes participantes $24 \%$ não estavam em educação, emprego, ou formação na idade de 19 anos, e 40\% das pessoas estavam com depressão e transtornos de ansiedade, e mais de $35 \%$ das pessoas com problema de uso de substâncias na idade de 18 anos (Mars et al., 2014).

Em relação aos transtornos mentais verificou-se que entre as meninas os problemas psicológicos e a automutilação podem entrar num círculo vicioso na forma de um sistema de feedback dinâmico tanto para o surgimento quanto para a estabilização de padrões patológicos. De maneira contrária, a relação problemas psicológico e automutilação entre os meninos não indica um problema igualmente sério, ou seja, embora a automutilação representa um sintoma de problemas psicológicos entre os meninos, ela pode não apresentar repercussões graves sobre desenvolvimento da saúde mental (Lundh et al., 2011). Os níveis de depressão, ansiedade, impulsividade e baixa autoestima foram associados com automutilação entre adolescentes de 15, 16 e 17 anos (McMahona et al., 2010).

Entre os fatores sociais e familiares, observou-se que as taxas de automutilação são mais elevadas entre os adolescentes que apresentaram preocupações recentes com a orientação sexual (Hawton et al., 2002) (McMahona et al., 2010).. Observou-se ainda associação entre automutilação deliberada no ano anterior entre as adolescentes que tiveram amigos ou familiares com história de automutilação, uso de drogas, depressão, ansiedade, impulsividade e baixa autoestima (Hawton et al., 2002). Entre os adolescentes do sexo masculino observa-se entre os fatores associados à automutilação deliberada no ano anterior a presença de amigos ou familiares com história de automutilação, uso de drogas e baixa autoestima (Hawton et al., 2002). A automutilação está evidenciada também entre meninas de 15-16 anos que vivem com um dos pais. (McMahona et al., 2010).
Entre os meninos com relato de vitimização verifica-se maior risco de automutilação quando há história anterior de automutilação, problemas com trabalhos escolares, abuso físico grave, e pensamentos de automutilação. De maneira diversa, observam-se entre os meninos sem relato de bullying maiores chances de automutilação quando há história de automutilação por um amigo ou familiar, pensamentos automutilação e consumo de drogas. (McMahona et al., 2010).

De acordo com Stallard, Spears, Montgomery, Phillips \& Sayal (2013) em estudo realizado com 2547 adolescentes com idade entre 12-16 anos os pensamentos e atos autolesivos são evidentes, sendo que um em cada cinco adolescentes apresentou pensamentos e um em cada dez executou pelo menos um ato de automutilação no decorrer de um período de seis meses. Compreende que a automutilação não possui intencionalidade suicida, mas que corresponde com um fator preditor de comportamento suicida fututo. Na pesquisa de Hawton et al. (2002) entre os 13,2\% adolescentes de 15-16 anos que relatam automutilação deliberada na vida verifica-se que $45,0 \%$ disseram que queriam morrer. Entre adolescentes de 15-16 anos a ideação suicida (sem automutilação deliberada) no ano passado foi relatada por $15,0 \%$, sendo mais comum entre as meninas (Hawton et al., 2002). O que sugere maior foco em programas de identificação e prevenção de lesões autoprovocadas.

\section{Automutilação Deliberada na População Adulta}

Nesta revisão foram considerados dados referentes a adultos jovens os estudos realizados com universitários. Estudo de prevalência de autolesão entre universitários norte-americanos mostra que alunos de graduação $(7,9 \%)$ são mais propensos do que os alunos de pósgraduação $(6,0 \%)$ para relatar autolesão. Entre os universitários que relataram autolesão, os comportamentos mais frequentes foram feridas $(36,7 \%)$, bater a cabeça ou outras partes do corpo $(35,8 \%)$, perfuração $(20,7 \%)$, arranhão $(18,4 \%)$, mordida $(17,5 \%)$ e corte $(11,1 \%)$, sendo mais comum entre as mulheres o relato de bater a cabeça ou outras partes do corpo e perfurar-se (Gollust et al., 2008). Dentre os universitários com história de autolesão observa-se que $32,5 \%$ apresentam associação com transtorno depressivo, 25,9\% com transtorno alimentar, $16,6 \%$ com ansiedade e $11 \%$ relataram pensamentos suicidas durante as últimas 4 semanas. Sendo que a prevalência de pensamentos suicidas entre os universitários com história de autolesão foi maior entre os homens do que nas mulheres (Gollust et al., 2008). 
Aqueles que relataram autolesão foram mais propensos a relatar o consumo diário de cigarros e uso de maconha do que os seus pares sem história de autolesão. Os estudantes que relataram estar em um relacionamento foram mais propensos a autolesão do que os universitários solteiros. Estudantes homens bissexuais e mulheres lésbicas apresentaram mais relato de autolesão do que universitários heterossexuais (Gollust et al., 2008). Em relação à percepção de necessidade de ajuda observa-se que entre os universitários que relataram autolesão cerca da metade $(50,9 \%)$ percebiam necessidade de ajuda, 15,7\% usaram alguma medicação psiquiátrica e mais de $80 \%$ estiveram com um profissional de saúde no ano passado, ainda se encontra que $19,9 \%$ apresentam história de ter feito terapia e $25,9 \%$ algum diagnóstico de problema de saúde mental (Gollust et al., 2008).

O estudo de prevalência de autolesão entre universitários italianos mostra que 20,6\%relataram história de comportamento de auto dano pelo menos uma vez na vida. Em relação à frequência observa-se que 82,5\% referiram mais de um episódio, 31,6\% mais de 10 episódios, e 3,9\% mais de 100 episódios de comportamento de auto dano. Quanto às formas específicas observa-se que $61,3 \%$ fizeram uso de um único método e $38,7 \%$ de vários métodos. Verifica-se maior frequência do comportamento de auto dano referente à interferência na cicatrização de feridas $(35,6 \%)$, seguido por corte $(25,4 \%)$, mordida $(18,6 \%)$, escarificações coçar grave $(16,9 \%)$ e inserção de pinos, agulhas e grampos sob a pele (15,3\%) (Sarno et al., 2010).

Observa-se associação entre comportamento de auto dano recorrente e psicopatologia ou sofrimento psíquico em geral. No que diz respeito às associações entre comportamento de auto dano entre universitários e mecanismos de defesa há evidências de associação com mecanismos de defesa mal adaptativos, incluindo projeção, acting out, dissociação, repressão, fantasia, conversão e evitação sugerindo maior desajustamento entre indivíduos com comportamento de auto dano recorrente. Ainda identifica-se maior variedade de sintomas psiquiátrico entre os universitários que apresentarem comportamento de auto dano recorrente, sugerindo a presença de maior gama de psicopatologia (Sarno et al., 2010). Constata-se que pacientes adultos jovens e adultos possuem mais risco ao comportamento autolesivo quando em uso de inibidores seletivos de receptação (ISRS) do que com prescrição de antidepressivos tricíclicos (Martinez et al., 2005).
Estudo de revisão sobre fatores de risco e métodos de automutilação entre grupos de minoria étnica no Reino Unido mostra que as disputas interpessoais precipitando automutilação foram mais frequente entre imigrantes negros, sugerindo o estresse situacional como fator de risco para a ocorrência de atos impulsivos de automutilação entre os imigrantes, neste sentido a automutilação pode surgir em resposta à aflição interpessoal (Bhui et al., 2007).

Entre os imigrantes sul-asiáticos encontram-se as substâncias domésticas, tais como água sanitária, gás, carvão e medicamentos, as mais utilizadas, entre os índios ocidentais são os medicamentos psicotrópicos mais comumente utilizados. Mais de $70 \%$ dentre as mulheres asiáticas internadas em uma unidade de tratamento de queimaduras após tentativa de suicídio usaram parafina para atear fogo em si mesmo (Bhui et al., 2007).

Estudo de revisão mostra que é mais frequentemente mulheres utilizarem tratamento médico para a automutilação. Dados prospectivos apontam que desemprego e não ter parceiros como preditivo de recorrência de lesões autoprovocadas no prazo de 12 meses. Identifica-se associação entre automutilação e experiências traumáticas na infância, neste aspecto são fatores significativos: problemas psicológicos por parte de um dos pais, separação dos pais, e separação precoce ou prolongada de um dos pais. Ainda se observam experiências na infância de negligência emocional, abuso físico ou psicológico, especialmente abuso sexual, entre adolescentes e adultos com comportamento de automutilação (Fliege et al., 2009). Verifica-se maior vulnerabilidade para Automutilação deliberada entre adultos que apresentaram pelo menos um evento negativo grave vida negativo nos últimos 6 meses. Entre os eventos de vida relatados encontram-se dificuldades de relacionamento com parceiros, amigos, cônjuges e vizinhos, crises financeiras e história de transtorno mental (Ritchie et al., 2011). Inquérito-piloto de prevalência de automutilação deliberada entre a população adulta carcerária de duas prisões do Reino Unido mostra que 71\% cometiam automutilação, sendo que a maioria respondeu que foram tentativas de suicídio. Ainda observa-se que $48 \%$ dos que receberam ordem de prisão preventiva foram encaminhados para tratamento psiquiátrico devido a risco de auto ferimento (Wessely et al., 1996).

Estudo americano com presos do sexo masculino em tratamento psiquiátrico mostra que $60 \%$ apresentavam história ou ameaça de automutilação, sendo que $18 \%$ exibiram episódio de automutilação na prisão. 
Quanto às formas específicas verifica-se maior frequência do comportamento de automutilação não fatal (45\%), tentativa de enforcamento (34\%), overdose de droga (5\%) e outras automutilações como asfixia, afogamento, bater a cabeça ou saltar de lugares altos (16\%) (Young et al., 2006).

Pacientes homens com padrão de uso pesado de bebida alcoólica apresentam maior risco de suicídio e de comportamento de automutilação deliberada. Entre os fatores de risco mais comuns para o suicídio entre usuários com padrão de uso pesado de bebida alcoólica encontram-se: sexo masculino, viver sozinho, desemprego, presença de pensamento e plano suicida, história de automutilação e de tratamento psiquiátrico (Taylor, Cooper \& Appleby, 1999).

Estudo realizado nas enfermarias de hospitais psiquiátricosde Londres mostra que a automutilação e o suicídio estão dentro dos incidentes mais frequentes, sendo que a automutilação se encontra associada com aumento da agressão física e ausência de pessoal (Bowers, Allan, Simpson, Nijman \& Warren, 2007).

Estudo com mulheres entre 18 e 45 anos recrutadas a partir de clínicas de tratamento comunitárias norteamericanas mostra que a intenção suicida e a letalidade da autolesão encontram-se associada com tentativa de suicídio. Observa-se associação da impulsividade de autolesão tanto na presença de tentativa de suicídio quanto comportamento de autolesão sem intenção suicida (Chapman, Derbidge, Cooney, Hong \& Linehani, 2009).

\section{Automutilação Deliberada na População Idosa}

A Demência é o diagnóstico psiquiátrico mais comum associado com o comportamento de auto dano entre idosos institucionalizados. O comportamento de auto dano em adultos mais velhos está associado à baixa qualidade de vida e saúde física precária. Estudo de revisão mostra que idosos institucionalizados com demência apresentam comportamento de auto dano, sendo mais frequente o auto beliscar, arranhar e bater o próprio punho. Evidencias mostram que $26,8 \%$ dos idosos institucionalizados com idade média de 82,3 anos apresentam comportamento de auto dano indireto, como a recusa em comer (Mahgoub et al., 2011). Ao longo dos três anos foram identificados 99 episódios de automutilação deliberada envolvendo 84 pacientes com mais de 65 anos de idade encaminhados pelo departamento de acidente e emergência de hospitais do Reino Unido.
A análise dos casos mostra que a relação entre homens e mulheres foi de 1:1,4. A média de idade para os idosos foi 72,5 anos e para as idosas, 75,3 anos. Dezenove dos 34 idosos eram casados em comparação com 13 das 48 idosas (Lamprecht, Pakrasi, Gash \& Swann, 2005).

Em relação às características do comportamento de automutilação entre os idosos observam-se que 37\%apresentavam história anterior de automutilação deliberada, em 93\% dos episódios a overdose de comprimidos foi o método de escolha, 25\% estavam sob cuidados de serviços psiquiátricos na época do episódio, $32 \%$ teve contato anterior com serviços psiquiátricos e $58 \%$ viu seu médico clínico geral dentro de 4 semanas antes do episódio. Ainda se observa que 37\% tiveram depressão maior no momento da automutilação deliberada, 40\% sofria de uma doença debilitante, 10\% apresentavam dependência alcoólica (Lamprecht et al., 2005).

Entre 2006 e 2008 registraram-se 2.352 casos de automutilação deliberada entre pessoas com 55 anos ou mais atendidas em hospitais irlandeses, sendo a maioria por mulheres (56,7\%). O número médio de episódio por pessoa por ano foi de 1,22 (máximo $=13$ ). Apenas um episódio por ano foi encontrado em $85,4 \%$ dos pacientes. A taxa de automutilação deliberada anual ajustada por idade taxa foi de 67,4 por 100 mil habitantes para os homens enquanto a taxa feminina, de 83,4 por 100 mil habitantes, portanto $24 \%$ de aumento. A incidência de automutilação deliberada entre os homens foi de 3,5 vezes enquanto para as mulheres, a incidência foi de 14,0. Os métodos de suicídio mais comuns foram enforcamento (41\%) e afogamento (29\%) entre os homens e afogamento (39\%) e ingestão de medicamentos (24\%) entre as mulheres (Corcoran et al., 2010).

\section{CONCLUSÃO}

O conceito polissêmico do comportamento autolesivo comprova a complexidade da manifestação do fenômeno. Entretanto diferentes definições obstem a compreensão do comportamento, principalmente na prática profissional, tornando-se um fator limitante para seu estudo. Em relação aos fatores de risco para o comportamento autolesivo, observaram-se generalidades do comportamento presentes em todas as etapas do desenvolvimento humano, bem como fatores específicos que se apresentam particularmente em cada fase do ciclo vital e de acordo com especificidades de cada gênero. Ressalta-se também a necessidade da contribuição de estudos relacionados aos fatores protetivos do comportamento autolesivo. 
Dessa forma, evidencia-se a importância de novos estudos sobre o comportamento autolesivo em diferentes etapas do ciclo vital, de acordo com especificidades de gêneros e principalmente a comparecia de fatores protetivos para o fenômeno. Torna-se imprescindível também a compreensão do fato em diversos lócus, por exemplo, a Internet, uma vez que o comportamento manifesta-se principalmente na adolescência, sendo os jovens os maiores utilizadores de tal ferramenta, tornando-se fonte de estudos para melhor apreensão da sua influência.

\section{IMPLICAÇÕES PARA A PRÁTICA CLÍNICA}

A apropriação do conhecimento dos fatores de risco do comportamento autolesivo é importante para possibilitar que profissionais de saúde estejam capacitados para operar na prevenção e m

anejo do fenômeno. O presente estudo apresenta informações que propiciam o profissional na identificação de casos de automutilação em relação ao ciclo vital possibilitando o olhar para possíveis lócus de ocorrência do fenômeno. Ainda proporciona informações que podem auxiliar em ações de prevenção e também o manejo de casos clínicos que apresentem na prática.

\section{REFERÊNCIAS BIBLIOGRÁFICAS}

Bhui, K., McKenzie K., \& Rasul F. (2007). Rates, risk factors \& methods of self harm among minority ethnic groups in the UK: A systematic review. BioMed Central Public Health, 19, 7-336. doi: 10.1186/1471-2458-7-336

Bowers, L., Allan, T., Simpson, A., Nijman, H., \& Warren J. (2007). Adverse incidents, patient flow and nursing workforce variables on acute psychiatric wards: The Tompkins acute ward study. International Journal of Social Psychiatry, 53(1), 75-84.

Cedaro, J. J., \& Nascimento, J. P. G. (2013). Dor e gozo: Relatos de mulheres jovens sobre automutilações. Psicologia USP, 24(2), 203-223. doi: 10.1590/S010365642013000200002

Chapman, A. 1., Derbidge, C. M., Cooney, E., Hong, P. Y., \& Linehani, M. M. (2009). Temperament as a propective predictor of self-injury among patients with Borderline personality disorder. Journal of Personality Disorders, 23(2), 122-140.
Corcoran, P., Reulbach, U., Perry, I. J., \& Arensman, L. (2010). Suicide and deliberate self harm in older Irish adults. International Psychogeriatrics, 22(8), 13271336. doi: $10.1017 /$ S1041610210001377

Dinamarco, A. V. (2011). Análise exploratória sobre o sintoma de automutilação praticada com objetos cortantes e/ou perfurantes, através de relatos expostos na internet por um grupo brasileiro que se define como praticante de automutilação (Dissertação de Mestrado). Universidade de São Paulo, São Paulo.

Fliege, H., Lee, J. R., Grim, A., \& Klapp, B. F. (2009). Risk factors and correlates of deliberate self-harm behavior: A systematic review. Journal of Psychosomatic Research, 66, 477-493. doi: 10.1016/j.jpsychores.2008.10.013

Fisher, H. L., Moffitt, T. E., Houts, R. M., Belsky, D. W., Arseneault, L., \& Caspi, A. (2012). Bullying victimisation and risk of self harm in early adolescence: Longitudinal cohort study. British Medical Journal, 344, 2683. doi: 10.1136/bmj.e2683

Giusti, J. S. (2013). Automutilação: Características clínicas e comparação com pacientes com transtorno obsessivo compulsivo (Tese de Doutoramento). Universidade de São Paulo, São Paulo.

Gollust, S. E., Eisenberg, D., \& Golberstein, E. (2008). Prevalence and correlates of self-injury among university students. Journal of American College Health, 56(5), 491-498. doi: 10.3200/JACH.56.5.491-498

Gratz, K. L. (2001). Measurement of deliberate selfharm: Preliminary data on the deliberate self-harm inventory. Journal of Psychopathology Behavioral Assessment, 23(4), 253-261.

Hawton, K., Rodham, K., Evans, E., \& Weatherall, R. (2002). Deliberate self harm in adolescents: Self report survey in schools in England. British Medical Journal, 325, 1207-1211.

Laukkanen, E., Rissanen, M. L., Honkalampi, K. I., Kylma, J., Tolmunen, T., \& Hintikka, J. (2009). The prevalence of self-cutting and other self-harm among 13- to 18-year-old Finnish adolescents. Social Psychiatry and Psychiatric Epidemiology, 44, 23-28. 
Lamprecht, H. C., Pakrasi, S., Gash, A., \& Swann, A. G. (2005). Deliberate self-harm in older people revisited. International Journal Geriatric Psychiatry, 20, 10901096. doi: 10.1007/s00127-008-0398-x

Lloyd-Richardson, E. E., Perrine, N., Dierker, L., \& Kelley, L. L. (2007). Characteristics and functions of nonsuicidal self-injury in a community sample of adolescents. Psychological Medical, 37(8), 1183-1192.

Lundh, L. G., Wångby-lundh, M., \& Bjärehed, J. (2011). Deliberate self-harm and psychological problems in young adolescents: Evidence of a bidirectional relationship in girls. Scandinavian Journal Psychology, 52, 476483. doi: 10.1111/j.1467-9450.2011.00894.x

Mahgoub, N., Klimstra, S., Kotbi, N., \& Docherty, J. P. (2011). Self-injurious behavior in the nursing home setting. International Journal Geriatric Psychiatry, 26, 2730. doi: 10.1002 /gps. 2486

Mars, B., Heron, J., Crane, C., Hawton, K., Lewis, G., Macleod, J., Tilling, K., \& Gunnell, D. (2014). Clinical and social outcomes of adolescent self harm: Population based birth cohort study. British Medical Journal, 349, 5954. doi: 10.1136/bmj.g5954

Martinez, C., Rietbrock, S., Wise, L., Ashby, D., Chick, J., Moseley, J., Evans, S., \& Gunnell, D. (2005). Antidepressant treatment and the risk of fatal and non-fatal self harm in first episode depression: Nested case-control study. British Medical Journal, 330, 1-7.

McMahon, E. M., Reulbach, U., Keeley, H., Perry, I. J., \& Arensman, E. (2010). Bullying victimisation, self harm and associated factors in Irish adolescent boys. Social Science \& Medicine, 71, 1300-1307.

Montero, C. V., Torres, B. R. A., González, V. G., Rodríguez, O. D. J., \& Arcos, C. L. (2015). Internet and sexual risk behavior for HIV/AIDS in young people Internet $\mathrm{y}$ conducta sexual de riesgo para VIH/SIDA en jóvenes. Enfermeria Global, 14(2), 160-168.

Noll, J. G., Horowitz, L. A., Bonanno, G. A., Trickett, P. K., \& Putnam, F. W. (2003). Revictimization and selfharm in females who experienced childhood sexual abuse. Journal Interpersonal Violence, 18(12), 14521471. doi: $10.1177 / 0886260503258035$
Ritchie, C. W., King, M. B., Nolan, F., O’Connor, S., Evans, M., Toms, N., Kitchen, G., Evans, S., Bielawski, C., Lee, D., \& Blanchard, M. (2011). The association between personality disorder and an act of deliberate self harm in the older person. Internatinal Psychogeriatrics, 23(2), 299-307. doi: 10.1017/S1041610210001742

Ronka, A. R., Taanila, A., Koiranen, M., Sunnari, V., \& Rautio, A. (2013). Associations of deliberate self-harm with loneliness, self-rated health and life satisfaction in adolescence: Northern Finland Birth Cohort 1986 Study. International Journal of Circumpolar Health, 72, 21085. doi: 10.3402/ijch.v72i0.21085

Sarno, F., Madeddu, K. L., \& Gratz, K. L. (2010). Selfinjury, psychiatric symptoms, and defense mechanisms: Findings in an Italian nonclinical sample. European Psychiatry, 25, 136-145. doi: 10.1016/j.eurpsy.2009.05.007 Skegg, K. (2005). Self-harm. Lancet, 366,1471-1483. doi: 10.1016/S0140-6736(05)67600-3

Souza, M. T., Silva, M. D., \& Carvalho, R. (2010). Revisão integrativa: $\mathrm{O}$ que é e como fazer. Einstein, $8(1 \mathrm{Pt}$ 1), 102-106.

Stallard, P., Spears, M., Montgomery, A. A., Phillips, R., \& Sayal, K. (2013). Self-harm in young adolescents (12-16 years): Onset and short-term continuation in a community sample. BioMed Central Psychiatry, 13, 328. doi: 10.1186/1471-244X-13-328

Taylor, C., Cooper, J., \& Appleby, L. (1999). Is suicide risk taken seriously in heavy drinkers who harm themselves? Acta Psychiatrica Scandinavica, 100, 309-311.

Wessely, S., Akhurst, R., Brown, I., \& Moss, L. (1996). Deliberate self harm and the Probation Service; an overlooked public health problem? Journal of Public Health Medicine, 18( 2), 129-132.

Young, M. H., Justice, J. V., \& Erdberg, P. (2006). Risk of harm: Inmates who harm themselves while in prison psychiatric treatment. Journal of Forensic Sciences, 51(1), 156-162. doi: 10.1111/j.1556-4029.2005.00023.x 